\title{
LE POSITIVISME ENTRE LA RÉVOLUTION ET LA CONTRE-RÉVOLUTION : COMTE ET MAISTRE
}

A lire l'ouvrage de Joseph de Maistre qu'Auguste Comte a le plus souvent cité, $D u$ Pape (1819), on ne peut qu'être étonné par la fréquence de cette référence, dont le caractère paradoxal semble d'ailleurs approprié à un auteur entre tous paradoxal. Sans doute, on comprend, qu'en lisant sous la plume de J. de Maistre une phrase comme celle-ci : « Pour nous la primatie du Souverain Pontife est précisément ce que le système de Copernic est pour les astronomes " ${ }^{1}$, Comte en ait apprécié l'ironie calculée, qui semble par ailleurs avoir troublé Pie VII et son secrétaire d'État, le cardinal Valenti. Mais est-il permis d'aller plus loin, et d'admettre que Comte ait repris à "l'illustre de Maistre" (comme il l'appelle) des éléments théoriques essentiels, comme par exemple sa théorie de la souveraineté ? Cette question pourrait aussi être formulée de la manière suivante : en s'efforçant de réconcilier les termes alternatifs de l'ordre et du progrès, Comte se serait-il rallié, en contradiction avec ses propres antécédents, théoriques et pratiques, aux idéaux de la contrerévolution? Or un tel revirement, si effectivement il a eu lieu, n'aurait pu s'opérer qu'après coup, divisant et opposant à elle-même la démarche suivie par le fondateur de la philosophie positive.

Remarquons pour commencer que la reconnaissance par Comte, de « l'éminente supériorité philosophique " de J. de Maistre, selon une formule de la $46^{\circ}$ leçon du Cours ${ }^{2}$, est relativement tardive eu égard à la précocité de sa propre démarche philosophique. La première référence explicite à $\mathrm{J}$. de Maistre se trouve en effet dans la correspondance de Comte à la fin de l'année $1824^{3}$, donc postérieurement à la réédition,

1. Joseph de Maistre, Du pape, éds Jacques Lovic et Johannès Chetail, Genève, Droz, "Les classiques de la pensée politique ", 1966, I, chap. 6, p. 55.

2. Auguste СомтE, E Euvres, Paris, Anthropos, 1968-1971, t. IV, p. 143 (cité par la suite comme (Euvres).

3. ID., Lettre du 25 déc. 1824 à Valat, in A. ComTE, Correspondance générale et Confessions, Paris/La Haye, Ed. de l'E.H.E.S.S./ Mouton, 1973, t. I, p. 147.

Revue de synthèse : IV S. No 1, janv.-mars 1991. 
dans sa forme définitive, de l'opuscule fondamental. On sait que celui-ci se termine par un examen critique des auteurs qui ont précédé Comte sur le terrain d'une synthèse entre la science et la politique - il s'agit de Montesquieu, de Condorcet et des Idéologues -, où aucune place n'est faite aux représentants de "l'école rétrograde ". Lorsque Comte reprendra cet examen en 1842, dans la $47^{\mathfrak{e}}$ leçon du Cours, qui complète la liste de ces «sources " en y ajoutant les noms d'Aristote et de Bossuet et en réservant le cas d'Adam Smith, il ne fera pas davantage mention de l'œuvre de J. de Maistre.

Ceci semble confirmer que la reconnaissance théorique de J. de Maistre ne peut s'interpréter, de la part de Comte, en termes d'emprunt. Aussi bien, sur quoi cet emprunt aurait-il pu porter? La réponse vient d'elle-même : il devrait concerner la notion d'un pouvoir spirituel distinct du pouvoir temporel, thème auquel Comte, en 1825, donc postérieurement à sa découverte de J. de Maistre, consacrera spécialement ses Considérations sur le pouvoir spirituel, le cinquième de ses opuscules de jeunesse repris plus tard en appendice au tome IV du Système de politique positive $^{4}$. Sur ce point précis, le rapport du spirituel et du temporel, qu'est-ce qui aurait pu faire l'objet d'un emprunt?

Envisageons d'abord cette question du côté de Comte, en nous demandant si cette distinction est vraiment nouvelle pour lui au moment où il en développe l'idée dans son texte de 1825 . Or il faut remarquer qu'elle avait déjà été introduite bien plus tôt et que son usage constituait peutêtre même le véritable point de départ de toute la démarche théorique de Comte. Elle apparaissait comme cruciale dans le second de ses opuscules de jeunesse, la Sommaire appréciation du passé moderne de 1820 , où l'on trouve en note cette indication très importante, d'où découle le clivage entre la forme ancienne et la forme moderne de la société :

« La division de la société et de tout ce qui la concerne en temporel et spirituel [...] est le perfectionnement le plus capital dans l'organisation sociale qui a été faite par les modernes. C'est là ce qui a primitivement fondé la possibilité de faire de la politique une science, en permettant de rendre la théorie distincte de la pratique. Seulement cette division, dans le nouveau système, n'est plus entre deux pouvoirs, mais entre deux capacités ${ }^{5}$.

La dernière formule de ce texte montre que cette idée venait directement de Saint-Simon, pour lequel avait été écrite la Sommaire apprécia-

4. Cf. CEurres, t. X, p. 176 sq. de l'Appendice.

5. Ibid., p. 7 de l'Appendice. 
tion, d'abord publiée sous sa signature dans L'Organisateur $^{6}$. Dans ses Lettres d'un habitant de Genève de 1803, Saint-Simon avait préconisé une réorganisation de la société sur le modèle de ce qu'on pourrait déjà appeler la cité scientifique, à partir de la dissociation entre des fonctions spirituelles, vouées à la considération de l'universel, et des fonctions temporelles, se consacrant à la gestion du particulier : la question est alors de savoir quelles sont les "capacités " en mesure d'assumer ces fonctions hétérogènes. On le voit, Comte, en lisant J. de Maistre, n'aurait pu y trouver tout au plus qu'une confirmation de cette orientation dont la perspective était d'emblée pour lui dessinée avec une particulière netteté.

Tournons-nous à présent du côté de $\mathrm{J}$. de Maistre pour envisager ce qui, dans son œuvre, aurait pu autoriser une telle confirmation. Il suffira pour cela de considérer la partie de cette œuvre que, à l'exclusion du reste (dans la $46^{\mathrm{e}}$ leçon du Cours sont dénoncées « l'inconséquence et la frivolité » des Considérations sur la France de 1796, l'ouvrage de J. de Maistre qui a aujourd'hui le mieux survécu), Comte a privilégiée: $D u$ Pape, publiée en 1819 et dont Comte ne semble avoir eu connaissance que cinq ans plus tard. Ce livre étonnant, déconcertant, développe une réflexion sur les problèmes généraux du "pouvoir", totalement détachée de la considération des capacités aptes à l'exercer, car celle-ci n'aurait pu que la relativiser. J. de Maistre affirme, en effet, le caractère absolu du pouvoir, considéré comme tel ; celui-ci se définit précisément par le fait qu'il n'est relatif à aucune condition : de ce point de vue, il est possible de parler, comme chez Bonald, d'un pouvoir en soi, de nature métaphysique, dont la forme lie le spirituel et le temporel par un rapport essentiel d'analogie qui les présente comme homogènes entre eux, à l'opposé de la conception défendue par Comte.

C'est pourquoi le principe de la souveraineté, tel qu'il s'incarne exemplairement d'après lui dans le dogme de l'infaillibilité pontificale, peut faire l'objet chez $\mathrm{J}$. de Maistre d'une déduction politique; cette déduction conclut, en quelque sorte a fortiori, du pouvoir temporel au pouvoir spirituel, ceux-ci étant liés entre eux par une correspondance réciproque. C'est dans ce sens qu'il écrit : "L'Église ne demande rien de plus que les autres souverainetés " ${ }^{7}$. Ce point a d'ailleurs été remarqué par Comte, comme le montre une réflexion insérée au début de la $46^{\mathrm{e}}$ leçon du Cours :

6. Le passage qui vient d'être cité d'après l'édition que Comte en a lui-même donnée se trouve également dans les ceuvres de Saint-Simon (Euvres de Cl.-H. de Saint-Simon, Paris, Anthropos, 1966, t. II, p. 85 de la deuxième partie reproduisant le volume IV de l'édition Dentu).

7. Cf. Du pape, op. cit. supra n. 1, I, chap. 19, p. 123. 
« L'illustre de Maistre [...] renonçant à tout appareil théologique, s'est efforcé dans son principal ouvrage de fonder le rétablissement de la suprématie papale sur de simples raisonnements historiques et politiques [...] au lieu de le commander directement de droit divin ${ }^{8}$.

Chez J. de Maistre apparait ainsi une image inattendue, parce que largement dé-théologisée, du théologique : ceci correspond parfaitement, du point de vue de Comte, à l'orientation essentielle du catholicisme, puisque avec celui-ci l'esprit religieux, suivant l'impulsion communiquée par sa forme ultime, le monothéisme, qui déjà l'incline vers la métaphysique, a pris une forme épurée, abstraite, transitoire entre deux états de la pensée.

Ceci signifie aussi que le raisonnement suivi par J. de Maistre maintient, dans la perspective analogique qui lui donne son contexte, une confusion permanente entre le spirituel et le temporel, ceux-ci ne pouvant jamais être véritablement dissociés. C'est dans cet esprit que J. de Maistre engage avec Bossuet une polémique sur la question du siège et de la personne, qu'il refuse de départager ${ }^{9}$, de même qu'il n'admet non plus aucun jeu entre une Église visible et une Église invisible. C'est en vain qu'on chercherait dans son livre l'amorce d'une réflexion sur les deux corps du roi, et à plus forte raison sur les deux corps du pape, réflexion à ses yeux inséparable de la conception formelle et juridique du pouvoir que précisément il récuse. Quelles que soient les formes et les conditions de son actualisation, le pouvoir est donc toujours et partout le même dans son principe substantiel ; ce principe ne peut être nullement divisé, la seule distinction qu'il supporte étant de degré, c'est-à-dire de puissance : en ce sens, ce qui, selon J. de Maistre, distingue le spirituel du temporel, ce n'est pas la nature du pouvoir, puisque celle-ci est par définition la même, mais c'est le fait que, dans le cas du spirituel identifié au religieux, cette nature soit élevée à la puissance, c'est-à-dire existe sous une forme " éminente ", qui en garantit la légitimité universelle.

$\mathrm{Si}$ on $\mathrm{y}$ réfléchit, cette argumentation est complètement différente, voire même opposée, à celle que développe Comte en insistant au contraire sur la dissociation et l'hétérogénéité des formes du pouvoir. Aussi bien, lorsqu'il évoque la discussion entre J. de Maistre et Bossuet, Comte renvoie ces deux auteurs dos à dos :

" On sait que de Maistre a reproché au grand Bossuet, et, à certains égards, avec raison surtout en ce qui concerne l'Église gallicane, d'avoir sérieuse-

8. Cf. Guvres, t. IV, p. 21.

9. Cf. Du pape, op. cit. supra n. 1, I, chap. 11, p. 75-81. 
ment méconnu la vraie nature politique du catholicisme; il ne serait pas difficile [...] de signaler aussi, chez le célèbre auteur du Pape, plusieurs inconséquences, sinon analogues du moins équivalentes. Et l'on prétendrait réorganiser les sociétés modernes d'après une théorie assez décrépite pour n'être plus depuis longtemps suffisamment comprise, même de ses plus illustres interprètes ! $\gg{ }^{10}$.

En ce qui concerne la question, pour lui essentielle, du pouvoir spirituel, Comte n'a pu reprendre à J. de Maistre aucun élément théorique tant les définitions de cette notion présentées par l'un et l'autre auteur s'inscrivent dans des contextes doctrinaux différents, et même opposés.

On peut ajouter cette remarque, dont le développement nécessiterait une autre étude : si Comte ne doit rien, ou très peu de choses, à Maistre qu'il ne cesse de citer, sa dette théorique est certainement fort importante à l'égard de l'autre grand représentant de l'école rétrograde auquel il ne se réfère pratiquement jamais de manière explicite : il avait certainement lu Bonald dès 1819, et il lui a repris les arguments essentiels sur lesquels il a appuyé sa propre critique de la psychologie ${ }^{11}$.

On ne peut alors éviter de poser une nouvelle question : la référence faite à J. de Maistre ne peut être sous-estimée ; mais, comme elle n'a pas valeur d'emprunt, que signifie-t-elle d'autre? Pour répondre, il faut partir de la longue note de la $46^{\mathrm{e}}$ leçon du Cours dans laquelle Comte s'est expliqué lui-même sur ce point :

« Profondément imbu, de bonne heure, comme je devais d'abord l'être, de l'esprit révolutionnaire, envisagé dans toute sa portée philosophique, je ne crains pas néanmoins d'avouer, avec une sincère reconnaissance, et sans encourir aucune juste accusation d'inconséquence, la salutaire influence que la philosophie catholique, malgré sa nature évidemment rétrograde, a ultérieurement exercée sur le développement normal de ma propre philosophie politique, surtout par le célèbre traité $D u$ Pape, non seulement en me facilitant, dans mes travaux historiques, une saine appréciation générale $\mathrm{du}$ Moyen Âge, mais même en fixant davantage mon attention directe sur des conditions d'ordinaire éminemment applicables à l'état social actuel, quoique conçues pour un autre état. Je crois, de même, avoir déjà suffisamment prouvé [...] que la politique positive peut être pleinement équitable envers la politique rétrograde et la politique révolutionnaire, sans leur faire aucune vaine concession de principes, et sans qu'une telle disposition nuise davantage à la fermeté de son langage qu'à la netteté de ses vues $»^{12}$.

10. Cf. Euvres, t. IV, p. 28.

11. A ce sujet, cf. Pierre Macherey, Comte, la philosophie et les sciences, Paris, Presses universitaires de France, "Philosophie ", 1989, p. 52-54 et ID., " Bonald et la philosophie ", Revue de synthèse, IV S., 1, janv.-mars 1987, p. 25-27.

12. Cf. CEurres, t. IV, p. 146. 
Il ne suffit donc pas d'évoquer la fascination qu'a pu exercer le style de pensée paradoxal de J. de Maistre, ni l'intérêt des renseignements qu'il a pu apporter à Comte concernant l'histoire et la doctrine du catholicisme. Il faut aller plus loin, et chercher à comprendre en quoi la philosophie positive a entrepris d'être «pleinement équitable envers la politique rétrograde et la politique révolutionnaire ».

Pour cela, on s'appuiera sur une hypothèse avancée par M. H. Gouhier : au moment où Comte a découvert J. de Maistre, il finissait, du moins le croyait-il, de se dégager de l'influence de Saint-Simon, et ainsi de Maistre a pu lui servir d'antidote, ou si l'on veut de réactif, intellectuel $^{13}$. En laissant pour le moment de côté la question de savoir si Comte a jamais pu échapper de manière définitive à l'inspiration qui lui avait été initialement communiquée par Saint-Simon, ce dont ferait douter la finale résurgence du thème religieux dans sa propre pensée, on doit reconnaître que l'hypothèse de $\mathrm{M}$. $\mathrm{H}$. Gouhier est féconde dans la mesure où elle dégage le caractère indirect du rapport entre ces deux auteurs; Comte semble s'être intéressé à $\mathrm{J}$. de Maistre, pour reprendre son propre langage, d'un point de vue davantage critique qu'organique : plutôt que de penser positivement quelque chose, il lui a permis de raisonner contre la réduction du spirituel au temporel qu'il imputait à l' « industrialisme » professé par Saint-Simon.

Peut-être faudrait-il même étendre le champ de cette hypothèse, en l'appliquant au rapport de la révolution et de la contre-révolution tel que l'interprète la philosophie positive. On en tirera l'indication que la pensée contre-révolutionnaire, dont J. de Maistre donnait l'illustration privilégiée, ne pouvait précisément de son point de vue avoir une signification " positive ". Entre les positions, alternatives en apparence, de la révolution et de la contre-révolution, on sait qu'il n'a pas pu être question pour Comte d'établir un équilibre ou un compromis, associant un minimum d'ordre à un minimum de progrès, alors que sa perspective était à l'inverse de multiplier l'un par l'autre, dans le sens d'un optimum. C'est bien pourquoi la confrontation entre révolution et contre-révolution, au lieu de conduire à une neutralisation de l'une par l'autre, reste pour lui tendanciellement polarisée, d'une manière qui privilégie nécessairement le point de vue de la révolution sur celui de la contre-révolution; cette dernière n'est justement, comme son nom l'indique, qu'une perspective contraire sur le mouvement irrésistible de la progression qu'elle accompagne, en la contestant, et dont elle dépend, même si c'est à son insu. Ceci suppose que Comte substitue à une conception abstraite et

13. Henri GouHier, La Jeunesse d'Auguste Comte, Paris, Vrin, 1941, t. III, p. 405. 
métaphysique de la révolution, présentée comme rupture artificielle du cours des choses, une conception organique, inspirée du modèle organique de la révolution astronomique, dont la progression est nécessairement continue.

En se fixant l'objectif de réconcilier ordre et progrès, Comte a-t-il voulu limiter négativement le mouvement de l'histoire, en le ramenant aux conditions restrictives d'une organisation préétablie, comme semblerait l'indiquer la thèse d'une subordination nécessaire du dynamique au statique ? Si cela était, on pourrait dire qu'il a adopté, au moins en partie, le point de vue d'une contre-révolution. Ou bien n'aurait-il pas plutôt, ce qui est tout autre chose, entrepris de régulariser le mouvement de cette progression, non en cherchant à la limiter en tant que telle, dans une perspective rétrograde, mais en contrôlant les excès, selon l'orientation fondamentale dessinée par la doctrine de la «perfectibilité »? On sait que, en lui retirant son caractère indéfini, Comte a repris ce dernier concept à Condorcet, avec tous les correctifs qu'il a pu lui apporter, qui constitue l'une des sources les plus authentiques de sa pensée.

Ainsi, par un retournement inattendu, le penseur contre-révolutionnaire assurerait dans la formation de la philosophie positive une fonction « critique », tandis que le penseur révolutionnaire jouerait à cet égard un rôle organique : cette hypothèse est confirmée par le fait que Comte ait traité de la Révolution française dans la $57^{\mathrm{e}}$ leçon du Cours, consacrée à l'étude de la phase positive de l'évolution humaine, et non, comme on aurait pu s'y attendre, dans la $55^{\mathrm{e}}$ leçon, qui concerne sa phase métaphysique et critique. De ce point de vue, l'image d'un Comte contrerévolutionnaire, telle qu'elle a pu être ici ou là propagée, serait une fiction, et celle-ci défigure l'esprit essentiel du positivisme.

Pierre Macherey, Université de Paris-I. 